\title{
Actitudes de estudiantes de primária en relación a las clases de Educación Física
}

CDD. 20.ed. 796.017

http://dx.doi.org/10.1590/1807-55092015000100127

\author{
Pedro GIL MADRONA* \\ Sheila Aparecida Pereira dos Santos SILVA** \\ Vicente ROMO PEREZ ${ }^{* * *}$ \\ Maria Luiza de Jesus MIRANDA**
}

*Faculdad de Ciencias de la Educación de Albacete, Universidad de Castilla La Mancha - Espanha.

**Universidade São Judas Tadeu.

***Faculdad de Ciencias de la Educación y del Deporte, Universidad de Vigo - Espanha.

\section{Resumen}

El objetivo de este estudio fue analizar las actitudes y reacciones emocionales de los estudiantes en el proceso de aprendizaje de la Educación Física. La muestra estuvo compuesta por 525 alumnos de $6^{\circ}$ curso de Educación Primaria de 20 colegios públicos de Albacete, España. Fue aplicado el cuestionario sobre el dominio afectivo (Physical Education Orientation Questionnaire - PEOO) con los resultados: a) no hubo diferencias significativas en la variable género; b) los alumnos aprecian la asignatura, aplican los conocimientos que en ella adquieren, conocen su valor y defienden su importancia, si bien dicha circunstancia queda lejos de alcanzar la máxima valoración. El estudio llega a la conclusion que la actitud de los estudiantes en relación a las clases de Educación Física fue positiva.

Palabras-Clave: Educación física; Aspectos afectivos; Actitudes; Emociones expresadas.

\section{Introducción}

Hace algunos años hemos experimentado un gran cambio en la educación pasando de un sistema educacional que tuvo como principal protagonista el profesor y asignando a lo cognitivo y memorístico la máxima consideración, a otro modelo que instala en el aula el uso de métodos activo-colaborativos, en que el propio alumno asume una alta responsabilidad en su proceso educativo. En este último modelo, el profesor cambia su rol tradicional de expositor $y$ trasmisor de conocimientos por el de estimulador y guía en el proceso de educativo ${ }^{1}$. Hoy dia es recomendable no enfocar solamente la enseñanza, sino enfocar lo que se espera que el alumno sea capaz de aprender, cómo son capaces de demostrar lo aprendido durante y al final del programa de instrucción, curso o etapa educativa. Para ello se utilizan enunciados denominados resultados de aprendizaje.

En este nuevo escenario, una nueva compreensión del alumno sobre la Educación Física (EF) es condición para que se genere una nueva actitud y comportamientos frente a las clases. Es esperado que el alumno aborde nuevos espacios, desafíos de aprendizaje que pueden ocasionar conflictos quedando en manos del profesor manejar las situaciones con el reto de evitar impactos negativos en el proceso educativo. En efecto, la calidad de las respuestas del alumno a los estímulos del profesor configura un ciclo que es particular para cada alumno y condiciona el progreso pedagógico de este. Sin duda en el éxito del proceso educativo, influyen el contexto familiar del alumno, su interés y voluntad en lograr los cambios perseguidos, y la calidad de los estímulos y respuestas presentes en el trascurso instructivo. De igual forma, un conjunto de sólidos valores, una adecuada formación pedagógica, y afecto por lo que hace, facilitarán al profesor su rol de motivador y guía.

MarChesi y Hernández ${ }^{2}$ señalan que los factores que mejor explican el fracaso académico son, por un lado, la falta de conocimientos y habilidades cognitivas y, por otro, la ausencia de motivación, interés y afectos positivos. En este sentido insisten en que la abundancia de fracasos en el aprendizaje en diversas edades y niveles educativos, puede ser explicada, en gran parte, por la aparición de actitudes negativas 
debidas a factores personales y ambientales, cuya detección será el primer paso para contrarrestar su influencia negativa con efectividad.

En este marco de necesidades y desafíos educativos, será sumamente importante entrelazar los resultados de aprendizaje con las actividades de enseñanza, aprendizaje y la apreciación o evaluación de estos, por lo que será preciso establecer los fines concretos que deseamos perseguir.

Estudios han confirmado que el dominio afectivo es factor determinante del éxito de los estudiantes en cualquier contexto educativo ${ }^{3-5}$.

Polya $^{6}, \mathrm{Vygotski}^{7}, \mathrm{Piaget}^{8}$, Vila y Callejo 9 , ERPIC et al. ${ }^{10}$ dan cuenta sobre la necesidad de considerar tanto los aspectos cognitivos como los afectivos y contextuales, en el proceso de enseñanzaaprendizaje en cualquier materia.

Hastie y Curtner-Smith ${ }^{11}$, Metzler $^{12}$, McMahon y MacPhaiL ${ }^{13}$ manifiestan que se deben conjugar los aspectos intelectuales y procedimentales con los emocionales, siendo estos últimos impulsores clave de la actividad en EF. Eso significa que, en ese proceso, se deben tomar en cuenta los conocimientos motrices de base y su didáctica, que incluye, entre otros aspectos, las estrategias, los recursos, la manera de transponer los conocimientos, las creencias y los afectos. Tales aspectos constituyen, por lo tanto, factores clave para determinar referentes capaces de dar cuenta del éxito o del fracaso no sólo de los alumnos y alumnas o de sus docentes, sino de, por ejemplo, las técnicas, los métodos y los recursos utilizados para enseñar o aprender determinados contenidos motrices. En efecto, tal y como plantea BISQUerra Alzina ${ }^{14}$, el desarrollo de la competencia emocional, considerada como una competencia básica para la vida, desemboca en la educación emocional. Por lo tanto hay que definir objetivos, asignar contenidos, planificar actividades, estrategias de intervención, para poder diseñar programas de intervención que van a ser aplicados y evaluados.

De ahí que nos hemos propuesto estudiar el dominio afectivo en concreto en un área predominantemente procedimental como es la EF ya que es en el campo afectivo que se puede buscar comprender la ansiedad de los alumnos, la frustración o inseguridad que sienten en determinados momentos y aumentar la autocompetencia motriz percibida por ellos. Además, la ausencia de motivación de los alumnos para las clases es un problema para los profesores de EF de todos los niveles. Sin duda, fomentar la motivación de los alumnos, promoviendo su interés y sentimientos de competencia ayudarán a alcanzar los objetivos de formación de hábitos y actitudes en esa materia ${ }^{15}$. Notase, en muchas ocasiones, que los alumnos tienen una imagen estereotipada que les hace tomar una determinada postura ante el aprendizaje de la $\mathrm{EF}^{16}$. Conocer mejor las representaciones del alumnado sobre las clases de EF permitirá que la planificación en esa área sea más eficiente, ayudando al profesor tener una clara dirección en la selección o diseño de los materiales educativos, los métodos, las actividades a realizar en la sala, gimnasio y eligir/ emplear instrumentos de evaluación de los resultados de aprendizaje.

Postulamos, por ejemplo, que es necesario trabajar para erradicar definitivamente el uso del temor como elemento de motivación, y con ello las barreras que interpone en el proceso educativo y, de esa forma, evitar posibles traumas que podrían afectar el comportamiento futuro del alumno en la materia. En esta línea VAN ACKER et al. ${ }^{17}$, bajo el prisma de la teoría social cognitiva, sostienen que los factores ambientales (contexto de la clase, su contenido, el comportamiento del profesor, etc.) influyen directa e indirectamente en los niveles de actividad física durante las clases de EF, así como en el interés de los alumnos por esta materia.

A tal efecto, apoyándonos en los planteamientos de la Taxonomía de Krathwohl et al. ${ }^{18}$, una de las pioneras en este campo, pero de vigente actualidad cuando se consideran las metas educacionales relacionadas a la clasificación allí propuesta, hemos intentado conocer la predisposición y los gustos de los alumnos y alumnas hacia sus clases.

Específicamente, examinamos las preguntas: ¿Cúal es la disposición en la que se hallan y se perciben los alumnos hacia la EF en relación con las dimensiones afectivas de Krathwohl? ¿Hay diferencias significativas en dicha disposición hacia la EF de los alumnos en función del género o nivel de estudios?

El objectivo de esa investigación fue analizar las actitudes y reacciones emocionales que experimentan los estudiantes de $6^{\circ}$ curso de Educación Primaria con el reto de conocer como las creencias, actitudes, y atribuciones positivas acerca de sí mismos como aprendices colaboran o constituyen una fuente de éxito en EF. La intención es colaborar para, desde ese conocimiento, diseñar y determinar con mayor precisión lo que los alumnos son capaces de aprender en EF y reducir las distancias entre lo que los docentes intentan enseñar y lo que de hecho sus alumnos aprenden. 


\section{Método}

\section{Participantes}

La población de estudio estuvo compuesta por 525 personas, $49,9 \%$ chicos y $50,1 \%$ chicas, edad média de 11 ańos, alumnos de $6^{\circ}$ curso de Educación Primaria de 22 colegios públicos de la provincia de Albacete, España, siendo ocho ubicados en el centro de Albacete Capital y catorce distribuidos por la provincia.

\section{Instrumento}

Se aplicó un cuestionario con 50 preguntas de Orientación en EF, basado en la Taxonomía de KRATHWOHL et al. ${ }^{18}$ (ANEXO I), dividido en cinco sub-escalas, con diez preguntas en cada una de ellas para analizar las dimensiones de Recibir, Responder, Valorar, Organización y Caracterización (Physical Education Orientation Questionnaire: PEOQ). Las cinco primeras preguntas en cada sub-escala tienen un valor positivo y las cinco últimas tienen un valor negativo.

Los 50 ítems están aleatoriamente asignados a lo largo del cuestionario elaborado de modo que guíe al alumno y evite respuestas preconcebidas. Los ítems se contestan usando la escala de tipo Likert con valoraciones del 1 - 5 (5. Casi siempre; 4. La mayoría del tiempo; 3. Algunas veces; 2. Casi nunca; 1 . Raramente) generando puntuaciones de 10 a 50 puntos en cada sub-escala. Esas pontuaciones se mantienen las mismas en todo el cuestionário.

\section{Procedimiento}

El estudio contó con la previa aprobación de los padres y madres de los participantes, así como con el permiso de los centros educativos. El cuestionario fue cumplimentado en clase de EF en el año de 2011, en presencia de un investigador que informó a los alumnos del objeto de la investigación, así como de la voluntariedad para participar y el anonimato del cuestionario. La cumplimentación se llevó a cabo en un tiempo aproximado de 20 minutos.

\section{Analisis de datos}

Se realizó un análisis descriptivo de la variable edad y de las cinco dimensiones del cuestionario, con error estándar $\left(\boldsymbol{s}_{\boldsymbol{*}}^{*}\right)$ e índices de confianza, desagregados por género. Los datos presentaron distribución normal. Se realizó un segundo análisis para comprobar la asociación entre los factores, utilizando el coeficiente de correlación de Pearson. En un tercer análisis, para la comparación de medias de las diferentes dimensiones entre chicos y chicas, si utilizó el test t-Student para grupos independientes, con nivel de significación $\mathrm{p}<0,05$. Los análisis estadísticos fueron realizados con el programa SPSS para Windows, version 20 (SPSS Inc, IBM, USA).

\section{Resultados}

En la TABLA 1 se presentan las medias de cada una de las dimensiones del cuestionario, de toda la muestra en general y desagregada por género.
La muestra está balanceada al estar los sujetos igualmente distribuidos en los dos grupos.

TABLA 1 - Estadística descritiva de los participantes y sus respuestas.

\begin{tabular}{lccccccccc}
\hline \multirow{2}{*}{ Variable } & \multicolumn{3}{c}{ Todos $(\mathbf{n}=\mathbf{5 2 5})$} & \multicolumn{3}{c}{ Chicos $(\mathbf{n}=\mathbf{2 6 2 , 4 9 , 9 \% )}$} & \multicolumn{3}{c}{ Chicas $(\mathbf{n}=\mathbf{2 6 3 , 5 0 , 1 \% )}$} \\
& Media & $\mathbf{s}_{\mathbf{x}}^{*}$ & $\mathbf{( 9 5 \% ~ I C )}$ & Media & $\mathbf{s}_{\mathbf{x}}^{*}$ & $\mathbf{( 9 5 \% ~ I C )}$ & Media & $\mathbf{s}_{\mathbf{x}}^{*}$ & $\mathbf{( 9 5 \% ~ I C )}$ \\
\hline Edad & 11,1 & 0,021 & $(11,0-11,1)$ & 11,1 & 0,032 & $(11,0-11,2)$ & 11,0 & 0,027 & $(10,9-11,1)$ \\
Recibir & 31,4 & 0,186 & $(31,0-31,8)$ & 31,3 & 0,268 & $(30,8-31,9)$ & 31,6 & 0,259 & $(31,1-32,1)$ \\
Responder & 30,2 & 0,158 & $(29,9-30,5)$ & 30,0 & 0,234 & $(29,5-30,5)$ & 30,4 & 0,215 & $(30,0-30,8)$ \\
Valorar & 29,3 & 0,235 & $(28,9-29,8)$ & 29,4 & 0,348 & $(28,7-30,1)$ & 29,3 & 0,316 & $(28,7-29,9)$ \\
Organización & 28,1 & 0,277 & $(27,6-28,6)$ & 28,5 & 0,382 & $(27,8-29,3)$ & 27,7 & 0,402 & $(26,9-28,5)$ \\
Caracterización & 31,8 & 0,247 & $(31,3-32,3)$ & 32,0 & 0,329 & $(31,3-32,7)$ & 31,6 & 0,367 & $(30,9-32,3)$ \\
\hline
\end{tabular}

$\mathbf{s .}_{\mathbf{x}}=$ Error Estándar; IC = Intervalo de Confianza. 
Atendiendo a la dimensión Recibir, podemos observar que tanto los alumnos como las alumnas solamente algunas veces tienen una aceptable "Disposición a prestar atención a un estímulo determinado". Sin embargo interesa que el educando adquiera la suficiente sensibilidad frente un estímulo o fenómeno presentado por el profesor, con el fin de provocar su participación. Esta categoría se descompone en tres subcategorías:

a) Toma de Conciencia, que tiene una clara connotación cognitiva. Apunta a conocer la importancia de un tema e implica el conocimiento y comprensión de las ventajas asociadas a una disposición favorable a recibir estímulos;

b) Disposición a recibir, el estado de expectativas de los alumnos por los estímulos que se generen en la clase. Dichos estímulos cada alumno los debe de percibir de manera personal y diferente, lo que condicionará su posición a aceptarlos. Ello implicará un compromiso personal que va mucho más allá de la toma de conciencia de la subcategoría anterior;

c) Atención controlada o selectiva, se refiere a la disposición personal del alumno para recibir ciertos estímulos frente a los cuales es sensible. A tal efecto, el profesor deberá hacer uso de esos estímulos selectivos, haciéndose cargo de la diversidad de personalidades en su curso.

Corfirmamos por tanto que en la práctica algunas veces existe un clima de respeto, cordialidad y permanente disponibilidad, lo cual contribuye a reducir el temor y las barreras que se generan, facilitando una interacción activa y colaborativa en la clase de EF, aunque sea posible desearse mejora en el dicho clima. El proceso formativo en y desde la EF debe de brindar oportunidades para compartir con los alumnos, como parte del programa volvido a desarrollar actitudes y habilidades sociales que ayudan a mantener comportamientos apropiados en la sociedad.

La dimensión Responder, que en el estudio obtiene una valoración muy similar a la categoría Recibir, tiene lugar en un momento en que ya está superada la resistencia a recibir estímulos. En este estado aparecen expectativas que se hacen presentes en las siguientes subcategorías:

a) Consentimiento en responder, que equivale a una decisión voluntaria del alumno en base a su convencimiento de que es conveniente esforzarse por responder a los estímulos;

b) Disposición a responder, donde se progresa hacia una respuesta autónoma a los estímulos. El reconocimiento de los logros fortalece esta disposición;

c) Satisfacción al responder, en donde el consentimiento progresa hacia un mejor grado de cumplimiento, a través de respuestas de mayor autonomía y la consecuente satisfacción derivada de las expresiones de reconocimiento.

Será determinante que el docente de EF focalice su atención y su trabajo en las clases de EF en esta tercera subcategoría para formar hábitos en los estudiantes.

La dimensión Valorar implica un alto grado de comprometerse o dedicarse a una actividad porque uno cree que merece la pena. Esta categoría implica un grado tal de internalización que la reiteración habitual de la conducta valorada como aceptable y digna de ser mantenida respondería a lo que generalmente se conoce como actitud. El individuo está motivado por el valor conscientemente admitido de los estímulos que orientan la conducta. Esta dimensión está conformada por tres subcategorías:

a) Aceptación de un valor donde el individuo está identificado ya, como portador de la creencia, el valor y la actitud hacia una determinada situación, objeto, persona;

b) Preferencia por un valor, en donde el alumno se halla suficientemente comprometido con un valor, para buscarlo, ejecutarlo o exigirlo;

c) Realización, en donde el sujeto manifiesta una profunda convicción en la aceptación de una conducta que considera valiosa y digna de ser imitada.

La dimensión Organización, que obtuvo la puntuación más baja, comprende la propia perspectiva de adoptar nuevos valores e integrar los nuevos valores en un sistema de valores existente de forma metódica. La conforman las subcategorías de Conceptualización de un valor y la Organización de un sistema de valores.

A medida que las experiencias del sujeto se lo permiten, su discernimiento y capacidad de valorar van ensamblando actitudes, intereses y valores en un sistema de jerarquías. Un nuevo valor, una nueva actitud sólo se incorporan en la medida en que el esquema aun se mantiene flexible.

La dimensión Caracterización, que obtiene una puntuación algo superior a la categoría Recibir, presupone la existencia de una organización de valores que el individuo ha internalizado de modo consciente y estable, proporcionándole al individuo su dominio. Ello supone construir un modo de vida alrededor de ciertos valores; estar predispuesto a comportarse de forma coherente con los valores ya interiorizados o ideales cristalizados.

En la TABLA 2 se presentan los coeficientes de correlación de Pearson entre las diferentes dimensiones del cuestionario. 
TABLA 2 - Matriz de correlacion para las dimensiones.

\begin{tabular}{lccccc}
\hline Subescala & Recibir & Responder & Valorar & Organización & Caracterización \\
\hline Recibir & --- & & & & \\
Responder & 0,39 & --- & & \\
Valorar & 0,33 & 0,29 & --- & & \\
Organización & 0,19 & 0,25 & 0,27 & 0,41 & -- \\
Caracterización & 0,22 & 0,26 & 0,25 & & \\
\hline
\end{tabular}

La relación entre ellas es baja, en ningún caso explican el $25 \%$ de la varianza $(r=0,5)$. Los factores que presentan una mayor correlación son Caracterización y Organización $(\mathrm{r}=0,41)$, y la correlación más baja es entre Organización y Recibir $(r=0,19)$.

Los datos resultantes de la comparación de medias de las diferentes dimensiones del cuestionario no presentan diferencias significativas en la variable género. Además, las médias y desviaciones estandar son similares en todas las dimensiones analizadas. Los p-valor resultantes de t-test son superiores al $5 \%$, permitiendo afirmar que no existen diferencias significativas entre los chicos y las chicas en ninguna de las variables analizadas (TABLA 3).

TABLA 3 - T-test de diferencias de género.

\begin{tabular}{|c|c|c|c|c|c|c|c|}
\hline \multirow{2}{*}{ Variable } & \multicolumn{2}{|c|}{ Chicos $(n=262)$} & \multicolumn{2}{|c|}{ Chicas $(n=263)$} & \multicolumn{2}{|c|}{ t-test } & \multirow{2}{*}{$\begin{array}{l}D E=\text { Desviación Estandar, } \\
t=T \text { de Student. }\end{array}$} \\
\hline & Media & DE & Media & DE & $\mathbf{t}$ & P-valor & \\
\hline Edad & 11,1 & 0,52 & 11,0 & 0,44 & 1,17 & 0,241 & \\
\hline Recibir & 31,3 & 4,36 & 31,6 & 4,19 & $-0,62$ & 0,537 & \\
\hline Responder & 30,0 & 3,80 & 30,4 & 3,48 & $-1,25$ & 0,210 & \\
\hline Valorar & 29,4 & 5,63 & 29,3 & 5,17 & 0,32 & 0,744 & \\
\hline Organización & 28,5 & 6,19 & 27,7 & 6,50 & 1,55 & 0,120 & \\
\hline Caracterización & 32,0 & 5,34 & 31,6 & 5,97 & 0,94 & 0,344 & \\
\hline
\end{tabular}

\section{Discusión}

El tema del dominio afectivo tiene una mayor implicación no solo por tomar parte em todos los procesos de aprendizaje, sino también porque los alumnos de Educación Primaria ponen en primer lugar, dentro de las distintas materias del curriculum escolar, su interés por las clases de $\mathrm{EF}^{19}$ que se relaciona con el deseo de aprender ${ }^{20-22}$. Desde la inserción de la EF en las escuelas y, en concreto, desde la promulgación de Ley Orgánica General del Sistema Educativo - LOGSE - en España, los argumentos de compromiso y disfrute se han convertido en puntos principales. Para rentabilizar socialmente este disfrute que sienten los alumnos por la EF, los maestros deben de ser sensibles respecto del afecto de los alumnos por la asignatura y proponer prácticas variadas, motivadoras y que proporcionen aprendizajes auténticos y efectivos ${ }^{23}$.
Si comparamos los datos de este estudio con los datos del trabajo que sirvió para validar el cuestionario $^{24}$ observamos que hay ciertas similitudes, ya que en ambos la dimensión Organización es la que más baja puntuación obtiene. No obstante, se encuentran algunas diferencias en los resultados obtenidos a favor del estudio de Faros y Shehu ${ }^{24}$. En nuestro estudio, los alumnos de $6^{\circ}$ y último curso de Educación Primaria mostraron clasificarse en dimensiones más bajas de la taxonomía y, por tanto, les cuesta más integrar e interiorizar dicha asignatura que a los alumnos del otro estudio, estudiantes de primer curso de Educación Secundaria, posiblemente por la diferencia de edad de un ańo y de un curso académico entre ambos grupos de alumnos. En ambos estudios, separados por la distancia, queda constatado que los alumnos aprecian la asignatura, 
aplican los conocimientos que en ella adquieren, conocen su valor y defienden su importancia.

Actitud favorável en relación a las clases de Educación Física también fue registrada en investigación de Betti y $\mathrm{Liz}^{25}$, por medio de cuestionario com cuestiones abiertas y cerradas, con un grupo de 151 niñas de $5^{\circ}$ a $8^{\circ}$. Año de la Educación Primária. Los componentes lúdicos, del movimiento, y las competiciones fueron los aspectos más valorados por ellas. La valoración de las competiciones también ocorrió en el estudio de Santos ${ }^{26}$, realizado em Portugal, desta vez por los niños. Alumnas del grupo estudiado por SAN$\operatorname{TOS}^{26}$ dicieron que les gustan las clases de Educación Física por sus aspectos sociales, por el divirtimiento que proporcionan y no tanto por las competiciones.

Las estudiantes brasileñas (64,9\% del grupo) eligieron la asignatura de Educación Física como su preferida, aunque no la juzguen más importante que Matemática, Portugués, Inglés, Ciencias o Geografia ${ }^{25}$.

Estudio cualitativo realizado por BegGiato y SiLva ${ }^{27}$, con 87 estudiantes brasileños del $6^{\circ}$. al $9^{\circ}$. Año de la Educación Primaria, evidencia que los alumnos aprecian las clases de Educación Física porque tienen la oportunidad de aprender sobre deportes, corroborando los estudios de BRANDÁO ${ }^{28}$ y de Betti y Liz ${ }^{25}$. Además, desarrollar su físico y sentirse bien son aspectos altamente valorados ${ }^{25,27}$.

Investigando la percepción de 84 alumnos de la Educación Secundária, en Brasil, sobre clases teóricas de Educación Física, Coelho y Portela ${ }^{29}$ corroboran los resultados de Beggiato y Silva ${ }^{27}$ y afirman que $95 \%$ de los estudiantes las valoraron positivamente, $90 \%$ de ellos nunca faltan a ese tipo de clase y el mismo número afirmó que ellas son importantes o muy importantes para sus vidas o, como apunta el estudio de BETTI y $\mathrm{LIz}^{25}$, para su desarrollo corporal.

Dos estudios realizados en Portugal ${ }^{26,28}$ llegaron a resultados semejantes. En el Concello da Maia, districto do Porto, 46 alumnos del $9^{\circ}$. Ano, em resposta a cuestionário de actitudes relativas à asignatura y a las unidades didácticas registraron actitudes positivas y consideraron sus contenidos importantes. En el Concello de Vila Nova de Gaia, entre 410 alumnos del $12^{\circ}$. Año de la Educación Secundária investigados, los alumnos del género masculino, en sus respostas a un cuestionário, manifestaron disposición favorable en relación a las clases de Educación Física y desear más tiempo de clase en comparación con las respuestas de las niñas ${ }^{28}$.

En general, la disposición frente a las clases merece más investigaciones explorando las diferencias entre géneros, una vez que el estudio de BrANDÃo ${ }^{28}$ destacó que obtener éxito en las actividades se muestra como un predictor significativo en el desarrollo de actitudes diferenciadas de niños y de niñas. BegGiato y SiLva ${ }^{27}$, por su turno, recomiendan que los profesores ofrezcan contenidos diversificados para atender a las diferencias de interés de los alumnos.

Esse estudio tuvo como objectivo analizar las actitudes y reacciones emocionales de los estudiantes en el proceso de aprendizaje de la Educación Física. La conclusion es que la actitud de los estudiantes en relación a las clases de Educación Física fue positiva.

Los resultados mostraron que no hubo diferencias significativas en la variable género; que, en general, los alumnos aprecian la asignatura, aplican los conocimientos que en ella adquieren, conocen su valor y defienden su importancia, aunque dicha circunstancia queda lejos de alcanzar la máxima valoración.

A la luz de los hallazgos de esta investigación cabría resaltar la importancia de los objetivos de dominio afectivo en la asignatura de EF y utilizar estrategias didácticas para guiar los aprendizajes en este ámbito educativo ya que parezce que los alumnos están mostrando una alta valoración de la asignatura, a pesar de que, a menudo los docentes dejan de volver su mirada para el objectivo de favorecer el aumento de interés del alumnado por la asignatura ${ }^{30} \mathrm{y}$, como advierten McMillan y $\mathrm{MaY}^{31}$, al hacer un resumen de trabajos de investigación sobre el dominio afectivo en relación con la enseñanza de las ciencias, la personalidad y la conducta del profesor son muy importantes en la formación de actitudes de los alumnos.

Tomando como apoyo investigaciones que examinan el dominio afectivo y su inflencia en la enseñanza/ aprendizaje de las matemáticas, ya que es un área en la que se han realizado más en este ámbito que en EF, posiblemente como consecuencia de que en la misma hay un alto porcentaje de alumnos que tienen dificultades para superar con éxito dicha materia ${ }^{32-34}$, nos es posible recomentar a los profesores de EF:

a) Que establezcan desde la primera clase un estilo de comunicación tranquila y directa, sin amenazas, a fin de lograr un clima de aceptación y confianza mutua alumno-profesor y profesor-alumno;

b) Que la cordialidad, respeto y exigencia se instale en las clases de EF como una mezcla adecuada para lograr el cumplimiento de las declaraciones y compromisos;

c) Que se incluya en los programas de la asignatura una unidad temática inicial destinada tanto a la comprensión de las competencias a desarrollar em términos afectivos, de valores y actitudinales, $\mathrm{y}$ 
los métodos a usar, incluyendo sus ventajas para un desarrollo autónomo;

d) Utilizar encuestas de autoevaluación de habilidades sociales y de los estados afectivos que faciliten la toma de conciencia sobre las carencias personales y grupales y un grado mayor de aceptación, da parte del alumnado, a los esfuerzos del profesor para reducirlas;

e) Que los alumnos tengan espacio para relatar sus vivencias traumáticas relacionadas a las clases de EF y los momentos de emoción positiva, para ayudar a la comprensión de todos sobre como experimentan las actividades propuestas; f) Que el profesor esté atento a la aceptación y manejo de las diferencias individuales para evitar conflictos derivados de ellas.

Y, de otro lado, en los planes de formación de los docentes de EF, convendría incluir:

a) El estudio, diseño y desarrollo práctico de la taxonomía de objetivos afectivos en la clase de EF;

b) Monitorización de la adquisición de las habilidades intrapersonales e interpersonales que inciden en el manejo del miedo, la ansiedad, el interés, la motivación, la curiosidad, el entusiasmo y el compromiso.

\title{
Nota
}

La Ley Orgánica General del Sistema Educativo (LOGSE), de 3 de octubre de 1990, publicada en el 4 de octubre, fue una ley educativa española, promulgada por el gobierno socialista y sustituyó a la Ley General de Educación de 1970. Ha sido derogada por la Ley Orgánica de Educación (LOE) en el año 2006.

\begin{abstract}
Attitudes of elementary school students in relation to Physical Education classes

The aim of this study was to analyze the attitudes and emotional reactions experienced by students in the learning process of physical education. The sample consisted of 525 students in 6th year of primary education than 20 public schools in Albacete, Spain. The questionnaire was applied on the affective domain (PEOQ - Physical Education Orientation Questionnaire) with the folowing results: a) not ocurred significant differences in the sex variable b) students appreciate the course, apply knowledge, know their value and defend its importance, although that fact is far from achieving the best possible estimate. The study comes to the conclusion that the attitude of the students in relation to the Physical Education is positive.
\end{abstract}

Key Words: Physical education; Affective aspects; Attitudes; Expressed emotion.

\section{Referencias}

1. Roque Vargas W. Pedagogía y curriculum: aspectos complementarios. Lima: Biblioteca Nacional del Perú; 2012.

2. Marchesi A, Hernández C, organizadores. El fracaso escolar: una perspectiva internacional. Madrid: Alianza; 2003.

3. Bandura A. Human agency in social cognitive theory. Am Psychol. 1989;44:1175-84.

4. Biggs JB, Moore PJ. Process of learning. New York: Prentice Hall; 1993.

5. Morgan P, Bourke S, Thompson K. The influence of personal school physical education experiences on non-specialist teacher's attitudes and beliefs about physical education. Annual Conference of the Australian Association for Research in Education; dec 2001; Fremantle, AU. Fremantle: AARE; 2001. [citado 10 set 2012]. Disponível em: http://www. aare.edu.au/data/publications/2001/mor01297.pdf.

6. Polya G. Cómo plantear y resolver problemas. México: Editorial Trillas; 1965.

7. Vygotski LS. El desarrollo de los procesos superiores. Furio S, tradutor. Barcelona: Editorial Crítica; 1979.

8. Piaget J. Inteligencia y afectividad. Dorin MS, tradutor. Buenos Aires: Editorial Aique, 2001. 
9. Vila A, Callejo M. Matemáticas para aprender a pensar: el papel de las creencias en la resolución de problemas. Madrid: Narcea Ediciones; 2004.

10. Erpic SC, Skof B, Boben D, Zabukovec V. Pupils's attitudes and motivation for physical education-some psychological aspects of physical education: pupils's attitudes and motivation. Int J Phys Educ. 2005;42:58-67.

11. Hastie P, Curtner-Smith M. Influence of a hybrid sport education-teaching games for understanding unit on one teacher and his students. Phys Educ Sport Pedag. 2006;11:1-27.

12. Metzler MW. Instructional models for physical education. Boston: Holcomb Hathaway; 2000.

13. McMahon E, MacPhail A. Learning to teach sport education: the experiences of a pre-service teacher. Eur Phys Educ Rev. 2007;13:229-46. [citado 10 set 2012]. Disponível em: : http://epe.sagepub.com/content/13/2/229.full.pdf+html.

14. Bisquerra Alzina R. Educación emocional y competencias básicas para la vida. [citado 10 set 2012]. Rev Invest Educ. 2003;21:7-43. Disponível em: http://www.doredin.mec.es/documentos/007200330493.pdf.

15. Scrabis-Fletcher K, Silverman S. Perception of competence in middle school physical education: instrument development and validation. Res Quart Exerc Sport. 2010;81:52-61.

16. Chen A, Ennis C. Goals, insterest, and learning in physical education. J Educ Res. 2004;97:329-38. [citado set 2012]. Disponível em: http://libres.uncg.edu/ir/uncg/f/C_Ennis_Goals_2004a.pdf.

17. Van Acker R, Carreiro da Costa F, De Bourdeaudhuij, Cardon G, Haerens L. Sex equity and physical activity levels in coeducational physical education: exploring the potential of modified game forms. Phys Educ Sport Pedag. 2010;15:159-73.

18. Krathwohl D, Bloom B, Masia B. Taxonomy of educational objectives. Handbook II: affective domain. New York: David McKay; 1956.

19. Gil Madrona P, Contreras Jordán OR. Interés y valoración del área de educación física por padres y alumnos en la enseñanza obligatoria. Rev Educ. 2003;332:327-55. [citado 10 set 2012]. Disponível em: http://www.educacion.gob. es/dctm/revista-de-educacion/articulosre332/re3321811443.pdf?documentId=0901e72b81256aef.

20. Almond L. Physical education in schools. London: Kogan Page; 1997.

21. Anderson LW, Bourke SF. Assessing affective characteristics in the schools. Mahwah: Lawrence Erlbaum; 2000.

22. Prusak KA, Treasure DC, Darst PW, Pangrazi RP. Effects of choice on the motivation of adolescent girls in physical education. J Teach Physl Educ. 2004;23:19-29.

23. Pieron M, Castro Girona MJ, González Valeiro MA. Actitudes y motivación en educación física escolar. Retos. 2006;10:5-22.

24. Faros AS, Shehu J. Minding the affective domain: an exploratory study of physical education orientation of junior secondary school students in Botswana. Int J Phys Educ. 2008;3:131-7.

25. Betti M, Liz MTF, Educação física escolar: a perspectiva de alunas do ensino fundamental. Motriz. 2003;9:135-42. Disponível em: http://www.rc.unesp.br/ib/efisica/motriz/09n3/08MBetti.pdf.

26. Santos FCM. Da importância dos conteúdos na atitude dos alunos face à aula de educação física [dissertação]. Porto (PT): Universidade do Porto, Faculdade de Ciências do Desporto e de Educação Física; 2001. Disponível em: http:// repositorio-aberto.up.pt/handle/10216/9846.

27. Beggiato CL, Silva SAPS. Educação física escolar no ciclo II do ensino fundamental: aspectos valorizados pelos alunos. Motriz. 2007;13:S29-S35. Disponível em: http://www.periodicos.rc.biblioteca.unesp.br/index.php/motriz/article/view/816/753.

28. Brandão DMR. Expectativas e importância atribuída à disciplina de educaçáo física: estudo comparativo por gênero nos alunos do $12^{\circ}$. ano de escolaridade nas escolas secundárias do Concelho de V.N. de Gaia [dissertação]. Porto (PT): Universidade do Porto, Faculdade de Ciências do Desporto e de Educação Física; 2002. Disponível em: http:// repositorio-aberto.up.pt/handle/10216/9840.

29. Coelho JMS, Portela BO. A visão discente a partir das aulas teóricas de educação física escolar. Col. Pesq Educ Fís. 2007;6:33-8. Disponível em: https://www.fontouraeditora.com.br/periodico/vol-6/Vol6n2-2007/Vol6n2-2007-pag33a38/Vol6n2-2007-pag-33a38.pdf.

30. Gil Madrona P, Cuevas R, Contreras OR, Díaz A. Educación física y hábitos de vida activa: percepciones de los adolescentes y relación con el abandono deportivo. Aula Abierta. 2012;3:115-24. [citado 10 set 2012]. Disponível em: http://www.uniovi.net/ICE/publicaciones/Aula_Abierta/numeros_anteriores/i20/14_AA_Vol.40_n.3.

31. McMillan JH, May MJ. A study of factors influencing attitudes toward science of junior high school students. J Res Sci Teach. 1979;16:217-22.

32. Debellis VA, Goldin GA. Affect and meta-affect in mathematical problem solving: a representational perspective. Educ Stud Math. 2006;63:131-47. [citado 10 set 2012]. Disponível em: http://deimos3.apple.com/WebObjects/Core.woa/ DownloadTrackPreview/tamu-public.2117699024.02117699032.2276558405.pdf. 
33. Gil Inácio N, Guerrero Barona E, Blanco Nieto L. El dominio afectivo en el aprendizaje de las matemáticas. Revista de Electrónica de Investigación Psicoeducativa. 2006; 4:47-72. [citado 10 set 2012]. Disponível em: http://www. investigacion-psicopedagogica.org/revista/new/ContadorArticulo.php?96.

34. Martínez Padrón O. Semblanzas de la línea de investigación: dominio afectivo en educación matemática. Paradigma. 2007;28:235-7. [citado 10 set 2012]. Disponível em: http://www.scielo.org.ve/pdf/pdg/v28n1/art12.pdf.

ANEXO 1 - Dominio afectivo. Cuestionario de Orientación en Educación Física.

Instrucciones: Por favor marque con un círculo el número que considere en cada afirmación

Claves:

5= Casi siempre estoy así

4= Estoy así la mayoría del tiempo

$3=$ Estoy así algunas veces

$2=$ Casi nunca estoy así

1= Raramente estoy así

\section{Sub-escala 1: Recibir}

\begin{tabular}{|r|l|l|l|l|l|l|}
\hline 1 & Asisto a las clases de educación física puntualmente & 5 & 4 & 3 & 2 & 1 \\
\hline 2 & Estoy concentrado durante las clases de educación física & 5 & 4 & 3 & 2 & 1 \\
\hline 3 & Sigo las instrucciones durante las clases de educación física & 5 & 4 & 3 & 2 & 1 \\
\hline 4 & Me fijo en la demostración del maestro durante las clases de educación física & 5 & 4 & 3 & 2 & 1 \\
\hline 5 & Estoy contento cuando llega la hora de educación física & 5 & 4 & 3 & 2 & 1 \\
\hline 6 & No me siento orgulloso vistiendo el atuendo de educación física & 5 & 4 & 3 & 2 & 1 \\
\hline 7 & Mi mente vaga durante las clases de educación física & 5 & 4 & 3 & 2 & 1 \\
\hline 8 & Busco excusas para escaquearme de la educación física & 5 & 4 & 3 & 2 & 1 \\
\hline 9 & No deseo gastar mis recursos en educación física & 5 & 4 & 3 & 2 & 1 \\
\hline 10 & Preferiría estar haciendo otra cosa que no fuera educación física & 5 & 4 & 3 & 2 & 1 \\
\hline
\end{tabular}

\section{Sub-escala 2: Responder}

\begin{tabular}{|l|l|l|l|l|l|l|}
\hline 11 & Me siento animado durante las clases de educación física & 5 & 4 & 3 & 2 & 1 \\
\hline 12 & Me siento bien siendo un estudiante de educación física & 5 & 4 & 3 & 2 & 1 \\
\hline 13 & Participo correctamente en las actividades de educación física & 5 & 4 & 3 & 2 & 1 \\
\hline 14 & Estoy en forma para las clases de educación física & 5 & 4 & 3 & 2 & 1 \\
\hline 15 & Disfruto durante las clases de educación física & 5 & 4 & 3 & 2 & 1 \\
\hline 16 & Estoy triste cuando se me asigna una tarea de educación física & 5 & 4 & 3 & 2 & 1 \\
\hline 17 & Me aburro cuando tomo parte en las actividades de educación física & 5 & 4 & 3 & 2 & 1 \\
\hline 18 & Me siento avergonzado siendo un estudiante de educación física & 5 & 4 & 3 & 2 & 1 \\
\hline 19 & Pongo poco esfuerzo en las tareas de educación física & 5 & 4 & 3 & 2 & 1 \\
\hline 20 & No disfruto practicando las habilidades aprendidas en educación física & 5 & 4 & 3 & 2 & 1 \\
\hline
\end{tabular}




\section{Sub-escala 3: Valorar}

\begin{tabular}{|l|l|l|l|l|l|l|}
\hline 21 & Comparto mis conocimientos de educación física con otros estudiantes & 5 & 4 & 3 & 2 & 1 \\
\hline 22 & Trabajo duro en cualquier tarea de educación física & 5 & 4 & 3 & 2 & 1 \\
\hline 23 & Me gustan los estudiantes que lo hacen muy bien en educación física & 5 & 4 & 3 & 2 & 1 \\
\hline 24 & Me tomo los deberes de educación física seriamente & 5 & 4 & 3 & 2 & 1 \\
\hline 26 & Me gusta compartir el equipamiento de educación física con los compañeros & 5 & 4 & 3 & 2 & 1 \\
\hline 27 & Creo que la educación física es una pérdida de tiempo & 5 & 4 & 3 & 2 & 1 \\
\hline 28 & No estoy seguro acerca del valor de la educación física & 5 & 4 & 3 & 2 & 1 \\
\hline 29 & Prefiero otras asignaturas a la educación física & 5 & 4 & 3 & 2 & 1 \\
\hline 30 & Creo que el tiempo dedicado a educación física es demasiado & 5 & 4 & 3 & 2 & 1 \\
\hline
\end{tabular}

\section{Sub-escala 4: Organización}

\begin{tabular}{|l|l|l|l|l|l|l|}
\hline 31 & Sugiero actividades cuando trabajamos en equipo durante las clases de E.F. & 5 & 4 & 3 & 2 & 1 \\
\hline 32 & Organizo mis proyectos de educación física & 5 & 4 & 3 & 2 & 1 \\
\hline 33 & Me enfado cuando la gente dice cosas negativas de la educación física & 5 & 4 & 3 & 2 & 1 \\
\hline 34 & Animo a mis compańeros de clase a que den lo mejor de sí en E.F. & 5 & 4 & 3 & 2 & 1 \\
\hline 35 & Odio las discusiones sobre la educación física & 5 & 4 & 3 & 2 & 1 \\
\hline 36 & Considero inadecuado debatir temas de educación física & 5 & 4 & 3 & 2 & 1 \\
\hline 37 & Me gusta que la educación física sea opcional & 5 & 4 & 3 & 2 & 1 \\
\hline 38 & Lamento haber elegido educación física como asignatura & 5 & 4 & 3 & 2 & 1 \\
\hline 39 & No me siento con confianza defendiendo el valor de la educación física & 5 & 4 & 3 & 2 & 1 \\
\hline 40 & Pienso negativamente sobre las oportunidades profesionales de la E.F. & 5 & 4 & 3 & 2 & 1 \\
\hline
\end{tabular}

\section{Caracterización}

\begin{tabular}{|r|l|l|l|l|l|l|}
\hline 41 & Me siento con confianza defendiendo la E.F. a estudiantes que no la cursan & 5 & 4 & 3 & 2 & 1 \\
\hline 42 & Busco oportunidades para practicar mis habilidades en educación física & 5 & 4 & 3 & 2 & 1 \\
\hline 43 & Intento mantener un alto nivel de rendimiento en educación física & 5 & 4 & 3 & 2 & 1 \\
\hline 44 & Defiendo la educación física contra críticas injustas & 5 & 4 & 3 & 2 & 1 \\
\hline 45 & Me preocupa hacerlo mal en educación física & 5 & 4 & 3 & 2 & 1 \\
\hline 46 & Tengo una actitud negativa hacia la educación física & 5 & 4 & 3 & 2 & 1 \\
\hline 47 & No estoy haciéndolo todo lo mejor que puedo en educación física & 5 & 4 & 3 & 2 & 1 \\
\hline 48 & Me siento inferior a los estudiantes que no cursan educación física & 5 & 4 & 3 & 2 & 1 \\
\hline 49 & Encuentro difícil aplicar las ideas de la educación física a mi estilo de vida & 5 & 4 & 3 & 2 & 1 \\
\hline 50 & Pienso positivamente acerca de hacer carrera en educación física & 5 & 4 & 3 & 2 & 1 \\
\hline
\end{tabular}
B. Datos personales
a. EDAD
b. GÉNERO
c. CURSO 

Coordenadoria de Educação Física Universidade São Judas Tadeu

R. Taquari, 546

03166-0oo - São Paulo - SP - BRASIL e-mail: sheila.silva@uol.com.br
Recebido para publicação: 08/03/2013

1a. Revisão: 19/04/2013

2a. Revisão: 25/09/2014

Aceito: 20/10/2014 